\title{
Energy from nuclear fission
}

\author{
M. RIPANI \\ INFN, Sezione di Genova - Genova, Italy
}

\begin{abstract}
Summary. - The physics of nuclear fission will be briefly illustrated, from the basic mechanism behind this phenomenon to the relevant physical quantities like nuclear cross sections, neutron flux and reaction products, together with the accompanying phenomenon of neutron capture and its role in determining how the fuel transforms in a nuclear reactor. The basic concepts underlying the operation of different types of nuclear reactors will be illustrated, along with the concept of fuel cycle. After touching on the aspect of safety, the current situation of nuclear power in the world, with its costs, its role in reducing carbon emissions, the available resources and finally the issues of waste management and accidents will be briefly illustrated.
\end{abstract}

\section{1. - Physics of fission}

What is fission and why can it produce energy? The basic reason is the behaviour of the nuclear mass and nuclear binding energy for different nuclear species. As is known from relativity, the mass of a bound system is given by the sum of the masses of its components, plus the binding energy of the system. The binding energy is by definition a negative quantity, i.e. the mass of the composite system is always less than the sum of the masses of its components. When applied to nuclear masses, this concept can be expressed through the equation

$$
M(Z, A)=Z M_{p}+(A-Z) M_{n}+B(Z, A)
$$




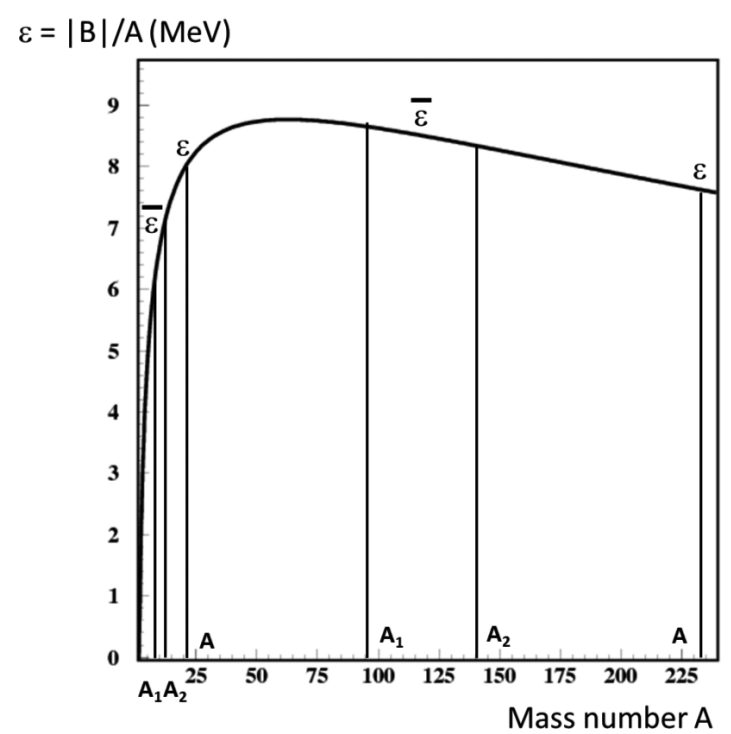

Fig. 1. - Absolute value of binding energy per nucleon, as a function of the mass number $A$.

where $M(Z, A)$ is the mass of a nucleus with $Z$ protons and $A$ protons and neutrons (i.e. $A-Z$ neutrons), $M_{p}$ is the proton mass, $M_{n}$ is the neutron mass and $B(Z, A)$ is the binding energy of the system, with $B(Z, A)<0$, i.e. $M(Z, A)<Z M_{p}+(A-Z) M_{n}$. $Z$ is called the atomic number, while $A$ is called the mass number. The absolute value of the binding energy is the energy that must be supplied from outside to overcome the binding nuclear force and set free all nucleons. We can also define the binding energy per nucleon, i.e. the average quantity of energy that must be supplied per single nucleon when separating all nucleons from one another. Figure 1 shows the absolute value of the binding energy per nucleon, $\epsilon=\frac{|B|}{A}$. It is seen that such value increases from the lightest nucleus, the deuteron, to the region of iron, then it decreases towards the heavier nuclei. In other words, iron is more bound than the light nuclei, while e.g. uranium is less bound than iron.

1`1. Energy balance. - Based on the above considerations, it is easy to see that a nuclear reaction where nuclei move from smaller to higher binding energy will release energy. Indeed, in going from the initial to the final state in the reaction, the total mass will decrease, so that the mass defect will be transformed to energy. Looking at fig. 1, it is clear that two light nuclei undergoing nuclear fusion, i.e. a nuclear system moving from the left-hand side to the right-hand side of the graph, will move towards higher binding, thereby releasing nuclear energy, i.e. giving rise to an exoenergetic nuclear reaction, a reaction which yields a net amount of energy. This is the mechanism at the basis of nuclear fusion [1], e.g. in the reaction ${ }^{2} \mathrm{H}+{ }^{3} \mathrm{H} \rightarrow{ }^{4} \mathrm{He}+n$, where about $17.5 \mathrm{MeV}$ are released in the process. On the other hand, heavy nuclei above iron can undergo fission, where the nuclear system splits into two nuclei, moving from the right-hand side to the 
left-hand side of the graph in fig. 1, again moving towards higher binding. This is called the process of nuclear fission and can also be exoenergetic. Let us consider in detail the energy balance of a fission process where a nucleus with $Z$ protons and $(A-Z)$ neutrons splits into two nuclei

$$
(Z, A) \rightarrow\left(Z_{1}, A_{1}\right)+\left(Z_{2}, A_{2}\right)
$$

where obviously $Z=Z_{1}+Z_{2}$ and $A=A_{1}+A_{2}$ and the masses of the nuclei are $M(Z, A)$, $M\left(Z_{1}, A_{1}\right)$ and $M\left(Z_{2}, A_{2}\right)$, respectively. The energy released in the reaction is called the $Q$-value and it is given by definition by

$$
Q=M(Z, A)-M\left(Z_{1}, A_{1}\right)-M\left(Z_{2}, A_{2}\right)
$$

By using eq. (1) we obtain

$$
\begin{array}{r}
Q=B(Z, A)-B\left(Z_{1}, A_{1}\right)-B\left(Z_{2}, A_{2}\right)= \\
-\epsilon A+\epsilon_{1} A_{1}+\epsilon_{2} A_{2}=-\epsilon A+\bar{\epsilon} A=(\bar{\epsilon}-\epsilon) A,
\end{array}
$$

where

$$
\bar{\epsilon}=\frac{\epsilon_{1} A_{1}+\epsilon_{2} A_{2}}{A_{1}+A_{2}} .
$$

Figure 1 tells us that if the initial nucleus is a heavy one (as indicated at the right of the picture), its fission into two medium-mass nuclei is such that $Q>0$. By repeating a similar calculation for two light nuclei undergoing fusion, it is easy to see that also in that case one can get $Q>0$.

The fact that fission of heavy nuclei is possible in terms of energy balance does not mean that fission will happen easily in a spontaneous manner. To understand that, imagine the two daughter nuclei (called fission fragments) being bound and merged together in a potential well (the basketball in fig. 2). In order to be separated from each other and transform into the smaller basketball and the tennis ball at the right of fig. 2, they will have to pass through the Coulomb repulsion barrier. This would be impossible in classical physics but it is possible in quantum mechanics, thanks to the quantum tunneling effect, which leads to the so-called spontaneous fission. The lifetime for the nucleus to decay via spontaneous fission will vary depending on the probability for the two nuclei to pass through the barrier. If energy is supplied to the initial heavy nucleus from the outside, the system will jump to a more excited quantum state, i.e. a higher energy state of the two fragments in the potential well, accompanied by a deformation of the nucleus schematically illustrated by the rugby ball in fig. 2 . Therefore the probability for the two fragments to penetrate the barrier will increase. This is what we call induced fission, which can actually happen with appreciable probability for nuclei with $A \gtrsim 230$.

How can we supply energy to the nucleus from the outside? For example, suppose that an incoming neutron is captured in the potential well of a heavy nucleus $(Z, A)$. Due 


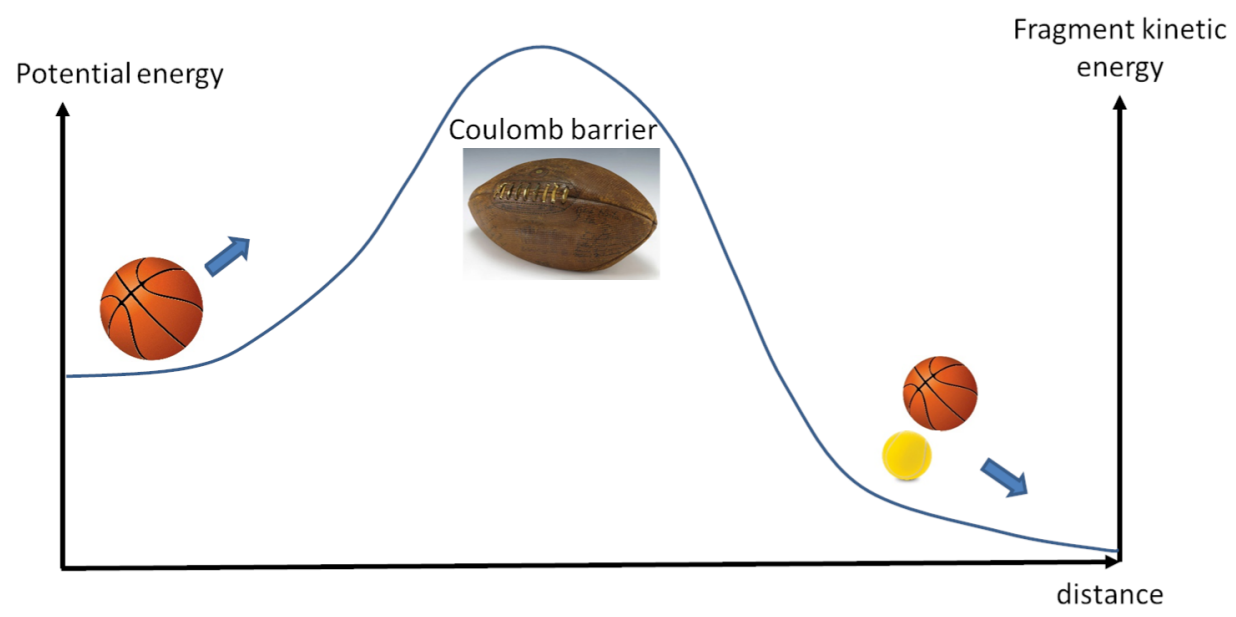

Fig. 2. - Nuclear potential energy as a function of the distance between the two fragments.

to the generally significant binding energy (a few $\mathrm{MeV}$ ) in the newly formed $(Z, A+1)$ nucleus, a few $\mathrm{MeV}$ of energy will be released and made available for the nucleus to approach the top of the barrier, thereby increasing the probability of the $(Z, A+1)$ nucleus to undergo fission by orders of magnitude. However, given the specific dependence of the binding energy on $Z$ and $A$, typically for odd- $A$ nuclei the binding energy of the extra neutron captured is higher than for even- $A$ nuclei. The former nuclei are called fissile and they can undergo fission with high probability even when capturing a very slow neutron (even for a neutron in thermal equilibrium with the surrounding medium). The latter nuclei are called fissionable and they can undergo fission with significant probability only when capturing a neutron with kinetic energy of the order of $1 \mathrm{MeV}$ or more. Two famous examples of the above are the fissile ${ }_{92}^{235} \mathrm{U}$ and the fissionable ${ }_{92}^{238} \mathrm{U}$ (see the following).

In practice, a chemical element of high importance in fission is uranium. Uranium is found in nature as two isotopes, ${ }^{238} \mathrm{U}$ and ${ }^{235} \mathrm{U}$, whose abundances are $0.7 \%$ and $99.3 \%$, respectively. While ${ }^{238} \mathrm{U}$ is fissionable, ${ }^{235} \mathrm{U}$ is fissile.

Fission is not the only exoenergetic nuclear reaction induced by neutrons. Just to make two examples, the reaction $n+{ }^{3} \mathrm{He} \rightarrow{ }^{3} \mathrm{H}+p$, when initiated by a very slow neutron (whose kinetic energy we can neglect), can release about $0.76 \mathrm{MeV}$ in form of the kinetic energy of the two final nuclei. The reaction $n+{ }^{6} \mathrm{Li} \rightarrow{ }^{3} \mathrm{H}+{ }^{4} \mathrm{He}$ can release about $4.78 \mathrm{MeV}$, again taken by the motion of the final nuclei. However, fission has a distinctive feature that makes it different from all others. Indeed, the fission process is accompanied by the emission of a few prompt neutrons, i.e. in addition to the splitting of the initial nucleus into two fragments, a few neutrons are emitted by the fragments in a very short time after the splitting. Such neutrons are fast, i.e. they have high speed and high kinetic energy (about $2 \mathrm{MeV}$ on average) and, as we will see shortly, if properly slowed down, can be very effective in inducing another fission, and so on and so forth. Therefore fission, under certain conditions, can give rise to a chain reaction, 
where the neutrons emitted by one fission can produce additional fissions, resulting in a very significant release of energy by the fissile material.

Another important process is radiative neutron capture, where the energy obtained from the capture of the neutron makes the resulting nucleus be in an excited state that then decays by emission of electromagnetic energy in form of energetic photons (gamma rays). This process can also occur on uranium nuclei and when fission is also possible it is a competitor process. Neutron capture can lead to the formation of radioactive nuclei. The case of radiative capture on ${ }^{238} \mathrm{U}$ is a special one, as it leads to the formation of plutonium, ${ }^{239} \mathrm{Pu}$, a fissile element not existing in nature.

1'2. Energy released and reaction products. - By calculating the specific mass balance given by eq. (3), it is easy to see that when a uranium nucleus fissions into two nuclear fragments, about $0.1 \%$ of uranium mass appears as fission energy of $\sim 200 \mathrm{MeV}$. Such an energy release is bigger than any other exoenergetic nuclear reaction by more than an order of magnitude (see for instance the above example of neutron capture on ${ }^{6} \mathrm{Li}$ ) and bigger than chemical reactions by several orders of magnitude (just for comparison, in the chemical reaction where a methane molecule is burnt, $\mathrm{CH}_{4}+2 \mathrm{O}_{2} \rightarrow \mathrm{CO}_{2}+2 \mathrm{H}_{2} \mathrm{O}$, about $9.2 \mathrm{eV}$ of energy are released, more than 7 orders of magnitude less). As anticipated above, the chain reaction is possible because along with the fission fragments an average of 2.5 prompt neutrons are emitted, with a mean kinetic energy per neutron of about $2 \mathrm{MeV}$. The average number of neutrons emitted is called $\nu$ and in general is of the order of $2-3$, depending on the nucleus undergoing fission and on the incident neutron energy. Out of the $200 \mathrm{MeV}$ released per fission, about $180 \mathrm{MeV}$ appear as kinetic energy of the emerging two nuclei, plus kinetic energy of the prompt neutrons, plus energy in form of prompt gamma rays (photons) emitted when nuclear fragments are produced in a nuclear excited state. Most nuclear fragments undergo $\beta$ decay, a much slower process in comparison to typical electromagnetic processes, caused by the nuclear weak force, where $Z$ of the nucleus increases by one unity, while $A$ stays the same, and concurrently an electron and an antineutrino are emitted. Therefore, due to the longer time scale of $\beta$ decay, additional energy is released later after fission and is carried by the electron, the associated antineutrino and possibly additional gammas. Antineutrinos escape from the reactor due to their negligible interaction with matter and do not deposit any energy in the reactor, while electrons and gammas do, contributing to the thermal budget of the reactor with about $13 \mathrm{MeV}$ per fission. When radioactive fragment build-up reaches a steady state, these $13 \mathrm{MeV}$ ( $6.5 \%$ of fission energy) contribute to the instant reactor thermal power. At the exact moment that the reactor is shutdown by stopping the chain reaction, the thermal power due to these radioactive decays, called residual heat, remains and decays slowly with time (see later).

1:3. Cross sections and flux. - The cross section is a physical quantity and an observable that characterizes a nuclear reaction (elastic, inelastic scattering, etc.). The cross section is connected to the range of the involved forces and represents the effective area of a nuclear target in a certain type of reaction or collision. Here we will only consider 
the so-called total cross section, defined as follows. Given a flux of particles incident on a single nucleus (target) per unit area $\mathrm{d} S$ per unit time

$$
\frac{\mathrm{d} N_{i n}}{\mathrm{~d} S \mathrm{~d} t}
$$

and given a reaction rate, which represents the number of interacting particles (scattered or absorbed projectiles) per unit time,

$$
R=\frac{\mathrm{d} N_{\text {reac }}}{\mathrm{d} t}
$$

then the total reaction cross section $\sigma$ is

$$
\sigma=\frac{\frac{\mathrm{d} N_{\text {reac }}}{\mathrm{d} t}}{\frac{\mathrm{d} N_{i n}}{\mathrm{~d} S \mathrm{~d} t}} .
$$

As is evident from the above equation, $\sigma$ has the physical dimensions of a surface and can be interpreted as an effective target area.

Given a macroscopic target comprising several nuclei with mass density $\rho$ and small thickness $x$, struck by a particle beam of intensity $I$ (particles/s)

$$
R=I \frac{\rho x}{A} N_{A} \sigma
$$

where $A$ is the target atomic weight e $N_{A}$ is the Avogadro number. For a target of arbitrary thickness, by dividing it in thin slices of thickness $\mathrm{d} x$, one obtains that

$$
I(x)=I(0) \exp \left\{-\frac{\rho}{A} N_{A} \sigma x\right\} .
$$

In nuclear physics, the cross section unit is the barn, corresponding to $10^{-24} \mathrm{~cm}^{2}$.

The quantity

$$
\Sigma=\frac{\rho}{A} N_{A} \sigma
$$

is called the macroscopic cross section and represents the probability of interaction per unit length of material crossed (and therefore has dimensions of the inverse of a length). It is easy to show that $1 / \Sigma$ is the mean free path of an incident particle in the material and $\Sigma v$ is the frequency at which a projectile with speed $v$ interacts with the crossed material.

Since the nuclear radius is roughly $10^{-12} \mathrm{~cm}$, the geometrical cross sectional area of the nucleus is roughly $10^{-24} \mathrm{~cm}=1$ barn. However, the combination of quantum mechanical and specific dynamical effects from the nuclear forces can make nuclear cross sections extremely variable over a few orders of magnitude. As an example, in fig. 3 are reported the cross sections for the processes of fission and radiative capture for the two uranium isotopes found in nature and used in nuclear reactors, ${ }^{238} \mathrm{U}$ and ${ }^{235} \mathrm{U}$, as a function of the 

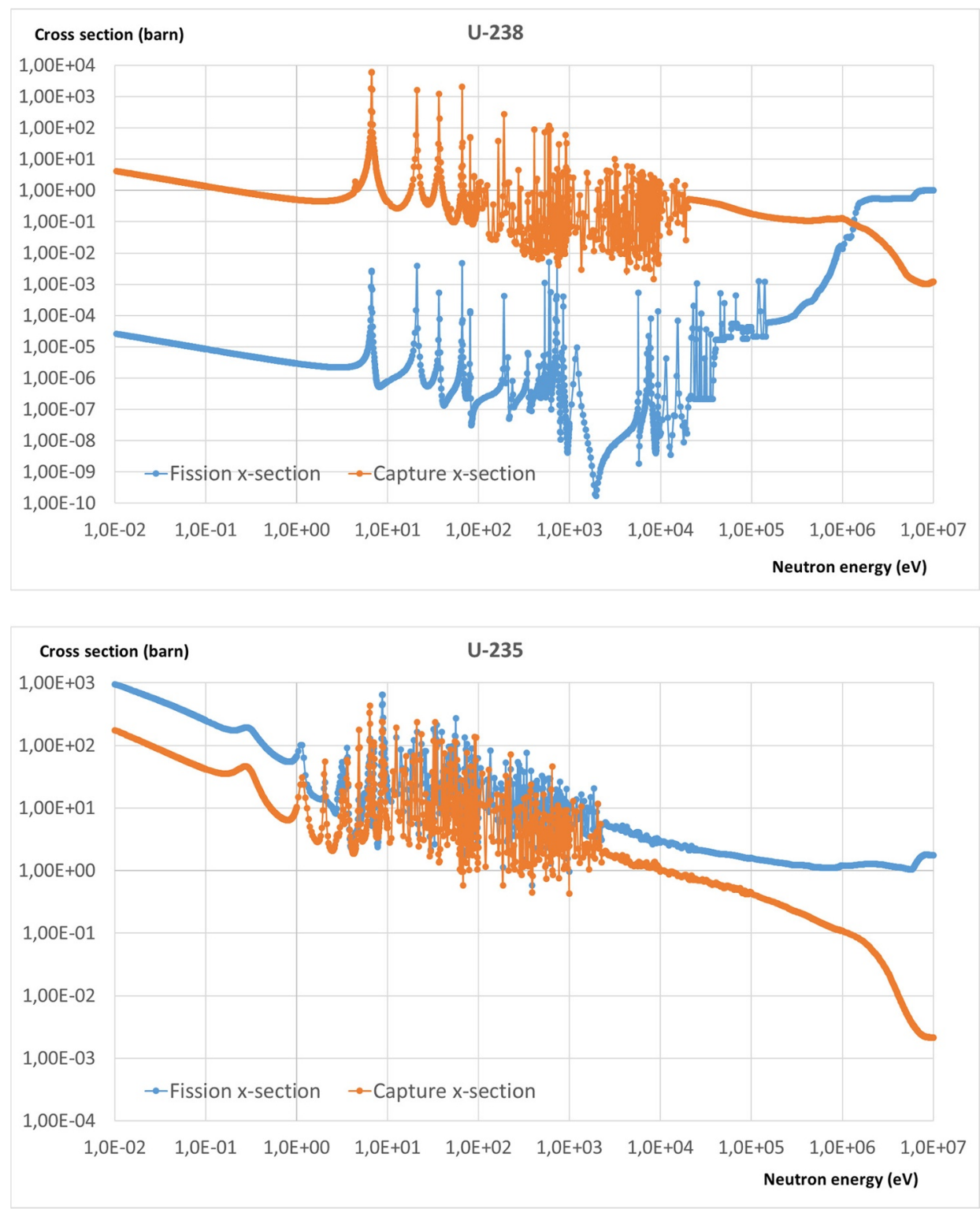

Fig. 3. - Fission (blue) and radiative capture (red) cross sections for the two isotopes of uranium.

neutron energy [2]. It is clearly seen that in ${ }^{235} \mathrm{U}$, for fast neutrons with kinetic energy around $100 \mathrm{keV}$ (or $0.1 \mathrm{MeV}$ ), both fission and radiative capture show a cross section in the order of the barn, but for lower energies the radiative capture for both nuclei and the fission for ${ }^{235} \mathrm{U}$ grow towards much higher values, following a $1 / v$ law. 
1*4. Neutron density and flux. - We define as neutron density the expected number of neutrons with energy between $E$ and $E+d E$, in the volume $d^{3} \vec{r}$ at the space position $\vec{r}$, at a time $t$ at a certain position and at a certain time in a nuclear assembly. This quantity is indicated by $n(\vec{r}, E, t)$ and can be measured for instance in neutrons per $\mathrm{cm}^{3}$ (or in $\mathrm{cm}^{-3}$ ). The reaction density $R(\vec{r}, E, t)$ is defined as the number of reactions in the volume $d^{3} \vec{r}$ about $\vec{r}$, at a time $t$, initiated by neutrons with energy between $E$ and $E+d E$ and is given by the equation

$$
R(\vec{r}, E, t)=n(\vec{r}, E, t) \Sigma v
$$

where again $v$ is the neutron speed. The reaction density is measured in reactions per $\mathrm{cm}^{3}$ per second. We give a special name to the quantity $n(\vec{r}, E, t) v$ : it is called the neutron "flux" $\phi(\vec{r}, E, t)$ and is measured in neutrons per $\mathrm{cm}^{2}$ per second. This quantity is essentially the number of neutrons arriving from whatever direction and crossing a unit surface in unit time (it may differ from other definitions of flux in physics but we will not discuss these differences here). Therefore, given a neutron flux $\phi$ and a macroscopic cross section $\Sigma$, the reaction density, number of reactions per unit volume, $R$ is simply given by $\Sigma \phi$. As an immediate example, consider a thermal reactor (see later) with $3 \mathrm{GW}$ thermal power $=3 \cdot 10^{9} \mathrm{~J} / \mathrm{s}$ (indicated by the symbol $3 \mathrm{GWth}$, with a $30 \%$ conversion efficiency it will produce about $1 \mathrm{GW}$ electric power, $1 \mathrm{GWe}$ ). Assume each fission releases order of $180 \mathrm{MeV}$ energy $=3 \cdot 10^{-11} \mathrm{~J}$ at the moment of fission. Then it follows immediately that in the whole reactor the fission rate is about $10^{20}$ fissions $/ \mathrm{s}$, which corresponds to $2-3 \cdot 10^{20}$ neutrons/s emitted and about $6 \cdot 10^{20}$ neutrinos/s from fragment $\beta$ decay. By using the known value of the fission cross section for thermal (very slow) neutrons on ${ }^{235} \mathrm{U}$, one can see that the flux inside the reactor must be in the order of $\phi \simeq 10^{13}-10^{14}$ neutrons $\mathrm{cm}^{-2} \mathrm{~s}^{-1}$.

15. Fuel. - We already encountered the definition of fissile (e.g., $\left.{ }^{233} \mathrm{U},{ }^{235} \mathrm{U},{ }^{239} \mathrm{Pu}\right)$ and fissionable (e.g., $\left.{ }^{238} \mathrm{U}\right)$ nuclei. There are also nuclei that can produce a fissile isotope via neutron radiative capture and subsequent $\beta$ decay. These nuclei are called fertile, meaning that they can be used to produce fuel and essentially comprise ${ }^{238} \mathrm{U},{ }^{232} \mathrm{Th}$. As an example, ${ }^{238} \mathrm{U}$ can produce a fissile nucleus via the chain of reactions

$$
n+{ }^{238} \mathrm{U} \rightarrow{ }^{239} \mathrm{U}+\gamma \rightarrow{ }^{239} \mathrm{~Np}+e^{-}+\bar{\nu} \rightarrow{ }^{239} \mathrm{Pu}+e^{-}+\bar{\nu}
$$

Natural uranium comprises $0.7 \%{ }^{235} \mathrm{U}$ and $99.3 \%{ }^{238} \mathrm{U}$, however reactors using mixed ${ }^{235} \mathrm{U}-{ }^{238} \mathrm{U}$ fuel typically need $3-5 \%{ }^{235} \mathrm{U}$, so that a procedure called uranium enrichment is needed to fabricate reactor fuel. The process of plutonium production, where actually a mixture of several $\mathrm{Pu}$ isotopes is produced, is called plutonium breeding. $\mathrm{Pu}$ breeding is important in cases where the used fuel is reprocessed to extract the plutonium isotopes. Under certain conditions, a reactor can produce more $\mathrm{Pu}$ than it consumes, in which case it is called breeder. From the cross sections shown in fig. 3 it follows that, in a reactor using a mixed ${ }^{235} \mathrm{U}_{-}{ }^{238} \mathrm{U}$ fuel and for neutron energies $<1 \mathrm{MeV},{ }^{235} \mathrm{U}$ provides 
the dominant contribution to the fission rate. It is worth mentioning that thorium, an abundant element in nature, can be used to produce a different type of fuel. Indeed, the reaction

$$
n+{ }^{232} \mathrm{Th} \rightarrow{ }^{233} \mathrm{Th}+\gamma \rightarrow{ }^{233} \mathrm{~Pa}+e^{-}+\bar{\nu} \rightarrow{ }^{233} \mathrm{U}+e^{-}+\bar{\nu}
$$

has as final product ${ }^{233} \mathrm{U}$, yet another fissile element. Use of thorium as a breeder of ${ }^{233} \mathrm{U}$ is studied as a possible way to increase the available stock of fissile elements. The whole process of mining, extraction of the uranium mineral from the ore, purification, fuel fabrication through enrichment, irradiation and final storage or disposal is called fuel cycle. In the open or once through fuel cycle the spent fuel is put in temporary storage waiting for final disposal. In the closed fuel cycle the spent fuel is reprocessed to extract the $\mathrm{Pu}$, which is then used to fabricate new fuel typically in the form of Mixed Oxide of $\mathrm{U}-\mathrm{Pu}(\mathrm{MOX})$.

16. Nuclear waste. - As discussed above, many of the fission fragments are radioactive, typically undergoing $\beta$ decay often accompanied by gamma emission. Such radioactive elements increase the radioactivity of the irradiated fuel, so that the fuel extracted from the reactor is a highly radioactive material that needs to be protected and shielded. While most of the radioactive fragments have relatively short half-lives, the longest being in the order of a few tens of years, a small group of fragments has very long half-lives in the order of $10^{5}$ years, the so-called Long-Lived Fission Products, LLFP. Also the aforementioned $\mathrm{Pu}$ production is one example of process leading to the appearance of Transuranics in the fuel, i.e. chemical elements beyond uranium that do not exist in nature. Reaction (13) leads to production of the ${ }^{239} \mathrm{Pu}$ isotope, but further neutron captures produce other isotopes ${ }^{240-242} \mathrm{Pu}$, of which ${ }^{241} \mathrm{Pu} \beta$-decays to americium, ${ }^{241} \mathrm{Am}$ and so on and so forth. Many of these Transuranics decay by $\alpha$ radioactivity and have long half-lives, from a few hundred years to a few hundred thousand years. Reprocessing of the irradiated fuel gives the possibility to recycle the $\mathrm{Pu}$ to fabricate new fuel, but whenever reprocessing is not applied or stopped, the resulting spent fuel is considered to be a nuclear waste or radioactive waste which needs to be properly stored and eventually disposed of. Indeed, such radioactive nuclei can be dangerous for the environment and for human health (due to direct exposure in the case of $\gamma$ but also $\beta$ radiation and due to ingestion or inhalation in the case of $\alpha$ and $\beta$ decay). Additional radioactive waste is produced in the reactor by neutron capture in the surrounding materials, producing activated materials with a varying degree of radioactivity. The International Atomic Energy Agency has come up with a classification scheme that is used as a template by each country to define categories of waste depending on its radioactivity, isotope content and possible heat production due to the radioactivity itself [3].

17. Fuel consumption. - Let's go back to our ideal $1 \mathrm{GWe}$ reactor burning only ${ }^{235} \mathrm{U}$. About $10^{20}$ fissions/s take place in the reactor, meaning that $10^{20}\left({ }^{235} \mathrm{U}\right.$ nuclei $) / \mathrm{s}$ disappear (actually somewhat more because of the concurrent radiative capture process). Therefore roughly $40 \mathrm{mg} / \mathrm{s}$ of ${ }^{235} \mathrm{U}$ are "burnt" in the reactor. For 1 year of operation 
at $80 \%$ load factor (the actual working time), this means a consumption of about 1 ton of ${ }^{235} \mathrm{U}$ : in volume of pure metallic ${ }^{235} \mathrm{U}$, this would be a cube of about $36 \mathrm{~cm}$ side. For comparison, the same amount of electric power is obtained by burning 1.6 Mtoe, million tons of oil equivalent (considering a slightly higher conversion efficiency of oil-fired power plants), or about 2 billion cubic meters of natural gas, or 2.2 million tons of coal. In a real fission reactor ${ }^{235} \mathrm{U}$ consumption is partly compensated by plutonium $\left({ }^{239} \mathrm{Pu}\right)$ breeding according to eq. (13), as ${ }^{239} \mathrm{Pu}$ is also a fissile nucleus. In practice, for an initial load of about 27 tons of enriched uranium, of which about $950 \mathrm{~kg}$ are ${ }^{235} \mathrm{U}$, after 1 year $280 \mathrm{~kg}$ of ${ }^{235} \mathrm{U}$ remain: about $560 \mathrm{~kg}$ of ${ }^{235} \mathrm{U}$ and $380 \mathrm{~kg}$ of other isotopes, mostly ${ }^{239} \mathrm{Pu}$ have been burnt, which is approximately the 1 ton we have been estimating above.

1'8. Fast and slow neutrons. - We have seen that the cross sections for nuclear reactions induced by neutrons depend a lot on the energies involved, therefore it is important to introduce some classifications of the neutrons according to their energies. It is customary to adopt the following classification: slow neutrons are those with kinetic energy $T_{n}<1 \mathrm{eV}$, in particular thermal neutrons at room temperature have $T_{n}$ around $0.025 \mathrm{eV}$ or $25 \mathrm{meV}$ (the value of $k T$, where $k$ is the Boltzmann constant and $T$ is the temperature of the system); epithermal neutrons are those with $1 \mathrm{eV}<T_{n}<100 \mathrm{keV}(0.1 \mathrm{MeV})$; finally, fast neutrons have $0.1 \mathrm{MeV}<T_{n}<20 \mathrm{MeV}$. Obviously neutrons in general can have energies above $20 \mathrm{MeV}$ but this is an extreme limit in reactor physics (e.g., neutrons from fusion of a deuteron and a tritium nucleus have $14 \mathrm{MeV}$ fixed energy).

It is easy to show in non-relativistic kinematics that after a scattering off a nucleus with mass number $A$, on average the kinetic energy of the neutron changes according to the ratio

$$
\frac{T_{n}^{\prime}}{T_{n}} \simeq \frac{1+A^{2}}{(1+A)^{2}}
$$

(where $T_{n}$ and $T_{n}^{\prime}$ are the kinetic energies before and after the scattering, respectively). For a heavy nucleus $A \gg 1$, one has that $T_{n}^{\prime} \simeq T_{n}$ or in other words, the neutron has to undergo many collisions in order to significantly lose energy. Consider instead the case $A=1$ (this is relevant for any medium containing hydrogen, where protons, hydrogen nuclei, will be among the targets). In this case $T_{n}^{\prime}=\frac{T_{n}}{2}$, i.e. on average a neutron will lose half of its energy at each collision and therefore few collisions are sufficient to rapidly decrease its energy. For collisions on a light nucleus like carbon $(A=12)$, the situation will be similar to that of hydrogen, although the average loss of energy per collision will be lower. Light materials containing hydrogen (water, paraffin, plastic materials in general etc.) or containing light nuclei (graphite, etc.) are called moderators, for their effectiveness in slowing down neutrons. In a thermal reactor, the fuel is immersed in water, which is used both as coolant and as moderator: the fast neutrons from fission will be slowed down very quickly via collisions with protons and oxygen nuclei in water and therefore they will have a very high fission cross section for ${ }^{235} \mathrm{U}$ (see fig. 3). 


\section{2. - Chain reaction and simple reactor kinetics}

For the reactor to work effectively and safely, the chain reaction must not diverge and must not die away. This means that, amongst the 2-3 neutrons emitted on average in the fission process, precisely one has to induce another fission event, not more, not less. The remaining fission neutrons will then either be absorbed by radiative capture or will leak out from the system. The character of the chain reaction depends essentially on the reactor composition and on its geometry and is defined through the quantity called $k$, also called multiplication coefficient

$$
k=\frac{\text { number of neutrons in one generation }}{\text { number of neutrons in the preceding generation }} .
$$

The condition $k=1$ corresponds to a critical reactor, i.e. a reactor actually working and producing thermal power from fission. For $k>1$ we have a supercritical reactor (fission reactions tend to runaway) and for $k<1$ we have a subcritical reactor (fission reactions tend to die away). In a "simple minded" reactor time evolution, called kinetics, it is relatively easy to show that the number of neutrons in the system evolves as

$$
n(t)=n(0) \exp \left(\frac{k-1}{\tau} t\right),
$$

where $n(0)$ is the initial number of neutrons and $\tau$ represents the average lifetime of a neutron in the system before it disappears due to fission, capture or escape (leakage). Here we clearly see that $k=1$ corresponds to a steady state, while $k>1$ implies that the system is diverging and $k<1$ corresponds to a decreasing population. Based on this simple kinetics, we see that the time constant governing the response of the reactor would be $\frac{\tau}{k-1}$. Since the typical neutron lifetime in a thermal power reactor is of the order of $10^{-4} \mathrm{~s}$, we see that even for $k=1.001$, the neutron population and therefore the reactor power would increase by a factor 2.7 in $0.1 \mathrm{~s}$, which would not be a manageable situation from a practical point of view. But there is one missing piece in the picture of fission discussed above. Actually, there is a small fraction (less than a percent) of neutrons originating from the fission process that are not emitted immediately, but some time later. These neutrons are emitted by highly excited fission fragments on a time scale from milliseconds to seconds and for this reason are called delayed neutrons. In simple terms, a nuclear reactor is made critical thanks to such small fraction of delayed neutrons and therefore they are the ones that dominate the reactor response time making it much longer, thereby allowing reactor control by neutron-absorbing control rods.

2*1. A bit more on neutron multiplication and transport. - By considering in more detail what processes can intervene along the route of the next-generation neutrons emitted after a single fission, one can come up with the following definition of multiplication coefficient $k$

$$
k=\eta f P_{N L},
$$




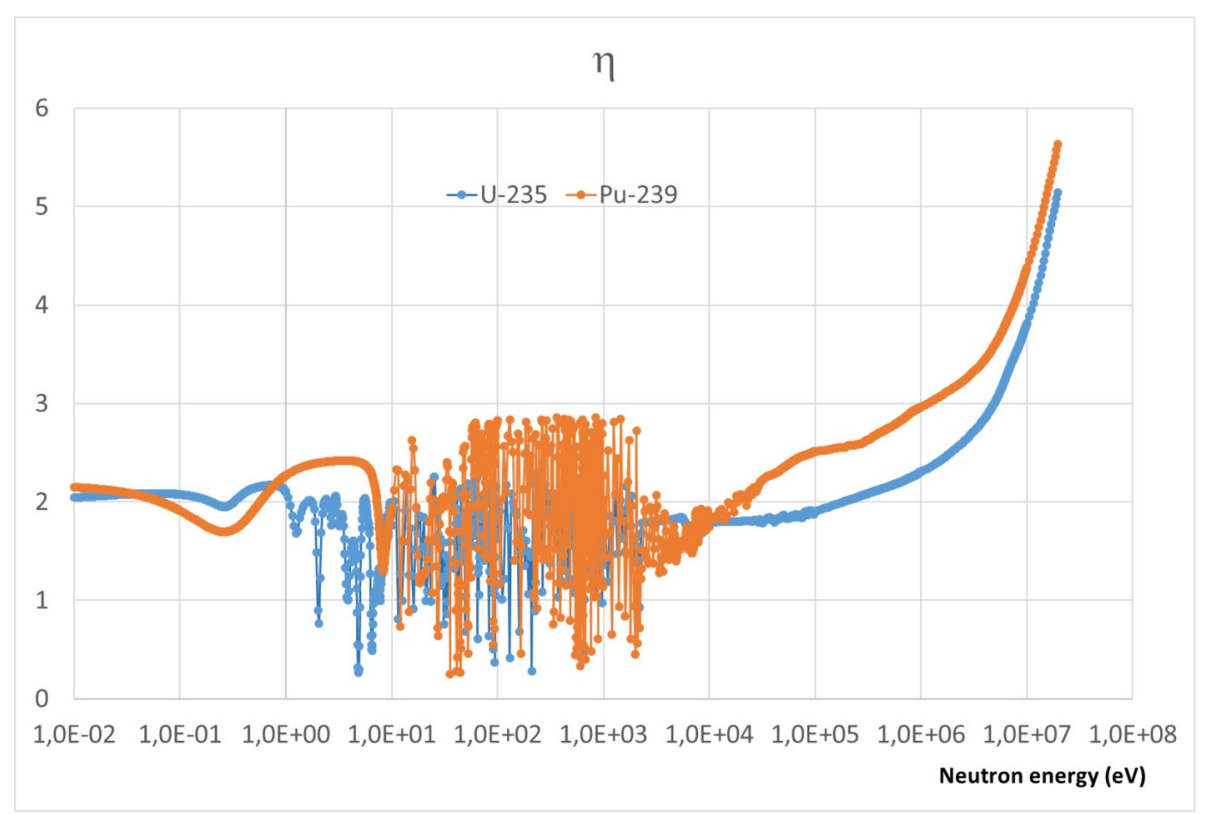

Fig. 4. - The parameter $\eta$ as a function of neutron kinetic energy for the two main fissile nuclei.

where $f$ is the conditional probability that, if a neutron will be absorbed, it will be absorbed in the fuel instead of structural elements; $\eta$ is the average number of neutrons produced per neutron absorbed in the fuel, in other words the average number of neutrons per fission $\nu$ multiplied by the probability that the absorption process leads to fission instead of radiative capture $\eta=\nu \frac{\sigma_{f}^{F}}{\sigma_{a}^{F}}$, where $\sigma_{a}^{F}$ is the absorption cross section in the fuel (absorption=fission+radiative capture) and $\sigma_{f}^{F}$ is the fission cross section in the fuel; finally, $P_{N L}$ is the probability that a neutron does not escape from the reactor (NoLeakage). For an infinite reactor clearly $P_{N L}=1$ and we get $k_{\infty}=\eta f$. In practice, an effective multiplication factor, $k_{\text {eff }}$ is defined, which takes into account neutron leakages and the reaction dependences on neutron energy. It is interesting to look at the behavior of the quantity $\eta$ as a function of neutron energy, shown in fig. 4. It is clear that for thermal neutrons, this number is around 2 , which means that effectively there are two neutrons available for further processes, one of which can trigger another fission, while another can be lost in captures or leak out of the system. In case of capture on a fertile nucleus like ${ }^{238} \mathrm{U}$, plutonium breeding can occur, but overall this will happen with relatively low probability. At the contrary, for high kinetic energies about $10-100 \mathrm{keV}$ (fast neutrons), $\eta$ becomes larger than 2 and so more neutrons will be available for breeding new fuel. Therefore, a so-called fast reactor, i.e. one in which neutrons are not moderated (see subsect. $3 \cdot 1$ ), may produce more plutonium than it consumes, in which case we will have a fast breeder. 


\section{3. - Main features of a nuclear fission reactor}

We saw that neutrons slow down when undergoing collisions with nuclei (in particular with light nuclei), therefore their energies in the core can go from about $10 \mathrm{MeV}$ (usually the maximum energy of fission neutrons), down to as low as $10^{-3} \mathrm{eV}$. We also saw that neutron cross sections have a strong dependence on neutron energy, in particular absorption cross sections such as capture or fission decrease very strongly with energy for particular species like ${ }^{235} \mathrm{U}$. When using fuels containing these particular species, it is easiest to maintain a fission chain reaction using slow neutrons. Hence most nuclear reactors until now (the so-called Generation I to III+) use low mass number materials such as water or graphite to slow down or moderate the fast fission neutrons, thereby slowing them down to energies comparable to the thermal energies of the nuclei in the reactor core. Therefore, by definition a thermal reactor is one where the average neutron energy is comparable to thermal energies (order of several tens of meV). Because of the very high fission cross section at thermal energies, this type of reactor requires the minimum amount of fissile material for fueling. As an example, a Light Water Reactor (LWR) can start with $3 \%{ }^{235} \mathrm{U}+97 \%{ }^{238} \mathrm{U}$ (a mixture enriched with respect to natural uranium). Burn-up (consumption) of ${ }^{235} \mathrm{U}$ is partly compensated by breeding of ${ }^{239} \mathrm{Pu}$ via neutron radiative capture and subsequent $\beta$ decays and after 1 year operation, the core may contain something like $1 \%{ }^{235} \mathrm{U}+1 \%{ }^{239} \mathrm{Pu}$. At the contrary, in a fast reactor the average neutron energies in the core are typically above $100 \mathrm{keV}$. As previously discussed, this can be accomplished if the core contains mostly high mass-number materials. This means that for core cooling the choice will be a liquid metal like sodium, lead or a lead-bismuth mixture, or alternatively the coolant should be a low-density material like Helium gas. On the other hand, $\sigma_{f}$ is smaller for fast neutrons, which means that a much higher percentage of fissile material is needed to sustain the chain reaction. In summary, the typical scheme of a thermal and a fast reactor are illustrated in fig. 5 (see also [4]).

3.1. Nuclear reactor types. - Most current reactors are based on fissile fuels, ${ }^{235} \mathrm{U}$ and ${ }^{239} \mathrm{Pu}$, with fission induced by slow neutrons at thermal energies. Ordinary water serves as both coolant and moderating material in the reactor. There are two major types of Light Water Reactors (LWR): Pressurized Water Reactors (PWR) and Boiling Water Reactors (BWR). In a PWR, the primary coolant is water maintained under very high pressure so that the coolant reaches a high temperature without steam formation within the reactor. Heat transported out of the reactor core by the primary coolant is then transferred to a secondary loop connected to a steam generator. In a BWR, the primary coolant water is maintained at lower pressure, so that boiling and steam formation occur within the reactor core itself, which serves directly as the steam generator, while secondary loop and heat exchanger are not present. In both PWR and BWR, the nuclear reactor itself and the primary coolant are contained in a large steel pressure vessel designed to accomodate the high pressures and temperatures. The heavy water $\left(\mathrm{D}_{2} \mathrm{O}\right)$ reactor takes advantage of the lower neutron capture cross section in deuteron with respect to hydrogen. This allows to have more neutrons around in the core and to 

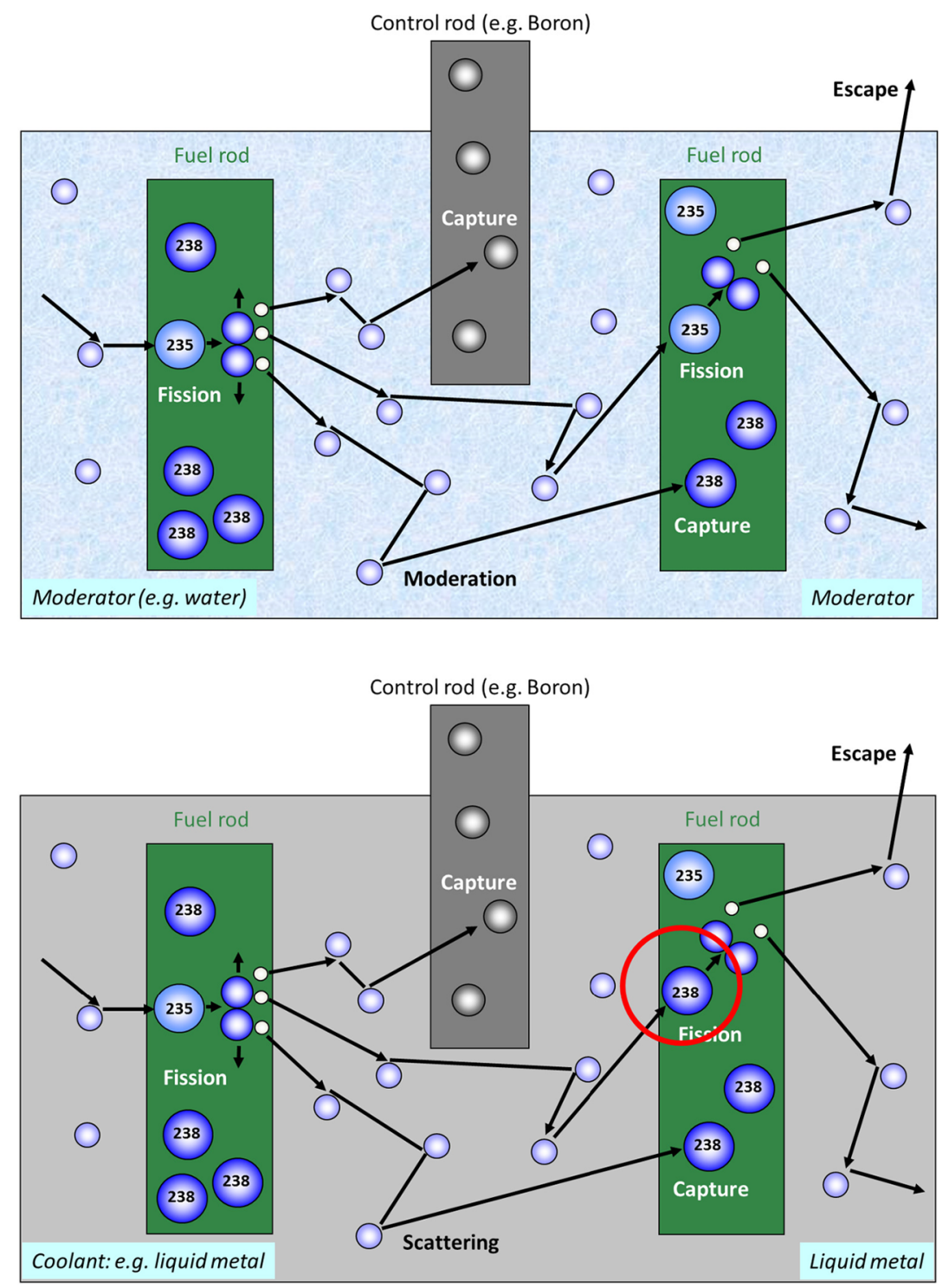

Fig. 5. - Conceptual schemes of thermal (top) and fast reactor (bottom).

use low-enrichment uranium fuels (including natural uranium). This concept has been developed for instance in Canada in the CANDU (CANadian Deuterium Uranium) series of power reactors. This type of reactors is indicated by the acronym PHWR (Pressurized Heavy Water Reactor). Yet another design is based on gas as coolant. For instance the early MAGNOX reactors developed in the UK used low-pressure $\mathrm{CO}_{2}$ as cooling gas. In general this reactor class is indicated by the acronym GCR (Gas Cooled Reactor). 
A more recent design is the High-Temperature Gas-cooled Reactor (HTGR, USA), that uses high-pressure helium as coolant. Gas-cooled reactors generally use graphite as moderator material to slow down neutrons to thermal energies. Finally, the Fast Breeder Reactor (FBR) does not use a moderator to work with fast neutrons (see sect. 2) and breeds more fuel than it consumes. Coolant is typically a liquid metal.

3.2. Decay heat. - Decay heat is the heat produced in the reactor core as a result of radioactive decay: the energy of the alpha, beta or gamma radiation is converted into atomic motion. Decay of the short-lived radioisotopes created by fission continues for some time after shut down. A practical approximation is given by the formula

$$
\frac{P}{P_{0}}=6.6 \cdot 10^{-2}\left[\frac{1}{\left(\tau-\tau_{s}\right)^{0.2}}-\frac{1}{\tau^{0.2}}\right] \text {, }
$$

where $P$ is the decay power, $P_{0}$ is the reactor power before shutdown, $\tau$ is the time since reactor startup and $\tau_{s}$ is the time of reactor shutdown measured from the time of startup (in seconds). At shutdown, the heat power is about $6.5 \%$ of the previous core power ( $\simeq 200 \mathrm{MWth}$ for a $1 \mathrm{GWe}$ reactor), which is sufficient to melt the core. About 1 hour after shutdown, the decay heat will be about $1.5 \%$ of the previous core power, after 1 day it will fall to $0.4 \%$ and after a week it will be $0.2 \%$, much smaller but still significant. For this reason, spent fuel rods are kept for long time in special cooling water pools, before being further processed. Removal of decay heat is therefore a very important aspect in designing a reactor and implementing safety measures, as the Fukushima accident has dramatically stressed.

3.3. Safety. - Safety is a very important aspect in nuclear power production. The core of a nuclear reactor produces radiation both while in operation and after shutdown, because of the high content of radioactive materials in the fuel and because of the neutron irradiation of materials in by the core and surrounding the core, which produces radioactive nuclei starting from stable ones. Therefore, the safety culture that permeates modern industry is an even more essential aspect of nuclear industry. Attention to safety is present from the design stage down to operation, shutdown and decommissioning of the plants and particular care is devoted to the analysis of possible accidental scenarios. A fundamental principle is the defence-in-depth, which means that a series of barriers, starting from the solid fuel form itself up to the reactor containment building, are put in place to contain radioactivity as much as possible at all times and in all instances. Obviously, in this respect, the role of independent regulatory authorities is crucial in overseeing all stages of a plant lifetime.

\section{4. - Fission energy in perspective}

4*1. Worldwide figures. - As illustrated in fig. 6, from the 1950s, when the first commercial reactors started to appear, until 1985 the number of operating reactors as well as the nuclear net generating capacity (i.e. the available power) has been increasing about 


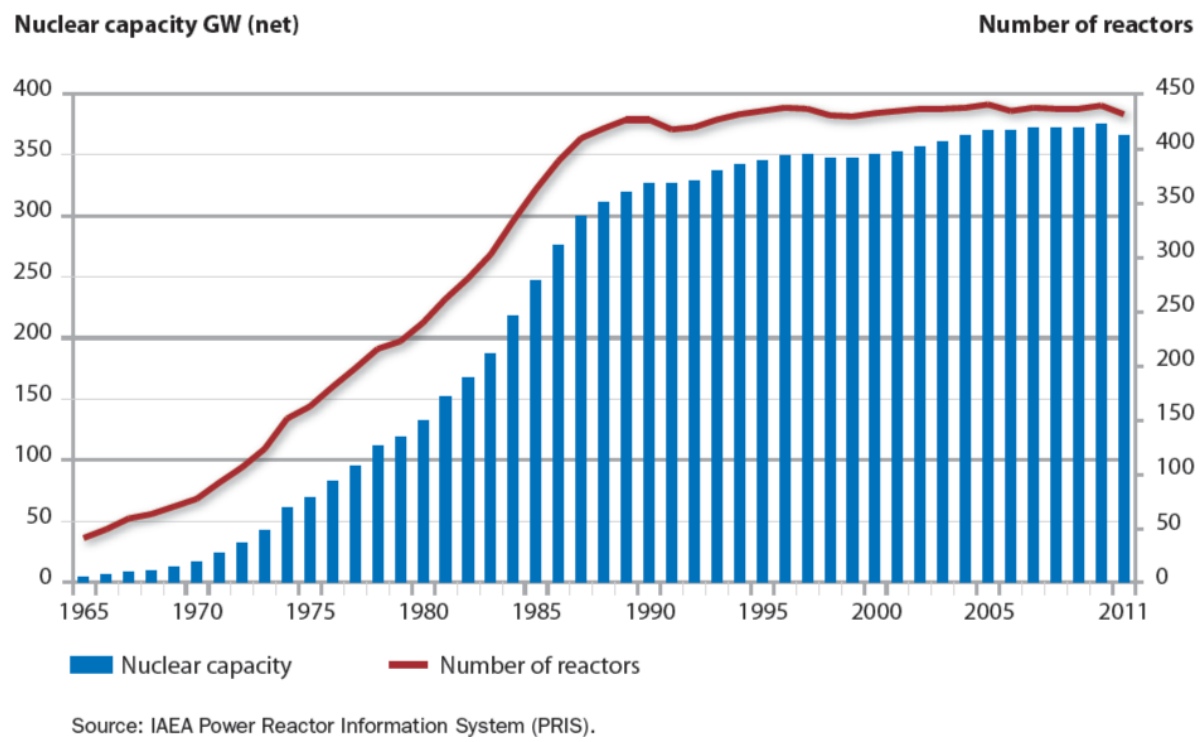

Fig. 6. - Worldwide nuclear generating capacity and number of operating reactors (1965-2011) [5] (C) OECD 2012).

linearly with time, then the number of reactor has remained approximately stable, while the generating capacity has been still increasing somewhat due to the improved efficiency of existing plants and some replacement of older installations [5]. As of October 2019, there were 449 reactors in operation or in long-term shutdown in the world, for a total net installed capacity of about $399 \mathrm{GWe}$, and 52 under construction, for a total capacity of about 53 GWe [6]. In fig. 7, the top and bottom panels show the number of reactors in operation and under construction in various countries, respectively. Figure 8 shows the share of nuclear power in electricity production as of October 2019 [6] country by country, while fig. 9 shows the share of nuclear power in the world electricity generation [7].

4'2. Cost of electricity and carbon emission scenarios. - The so-called Levelized Cost Of Electricity (LCOE) measures plant lifetime costs divided by energy production and it is typically given in USD/MWh, USD per MWh of energy produced. The LCOE calculates the present value of the total cost of building and operating a power plant over an assumed lifetime, so that it allows comparing different technologies with unequal life spans, project size, different capital cost, risk, return, and capacities. Especially for a low discount rate scenario, the nuclear LCOE appears to be competitive with other sources. Indeed, in the OECD study of 2015 [8], when considering a discount rate of $3 \%$ nuclear energy appears definitely cheaper than Coal and Combined Cycle Gas Turbines (CCGT), with a median of around 55 USD per MWh of energy produced, against almost 80 of coal and 100 of CCGT. When considering a $10 \%$ discount rate scenario, the nuclear LCOE median appears slight higher than Coal and CCGT, but a 


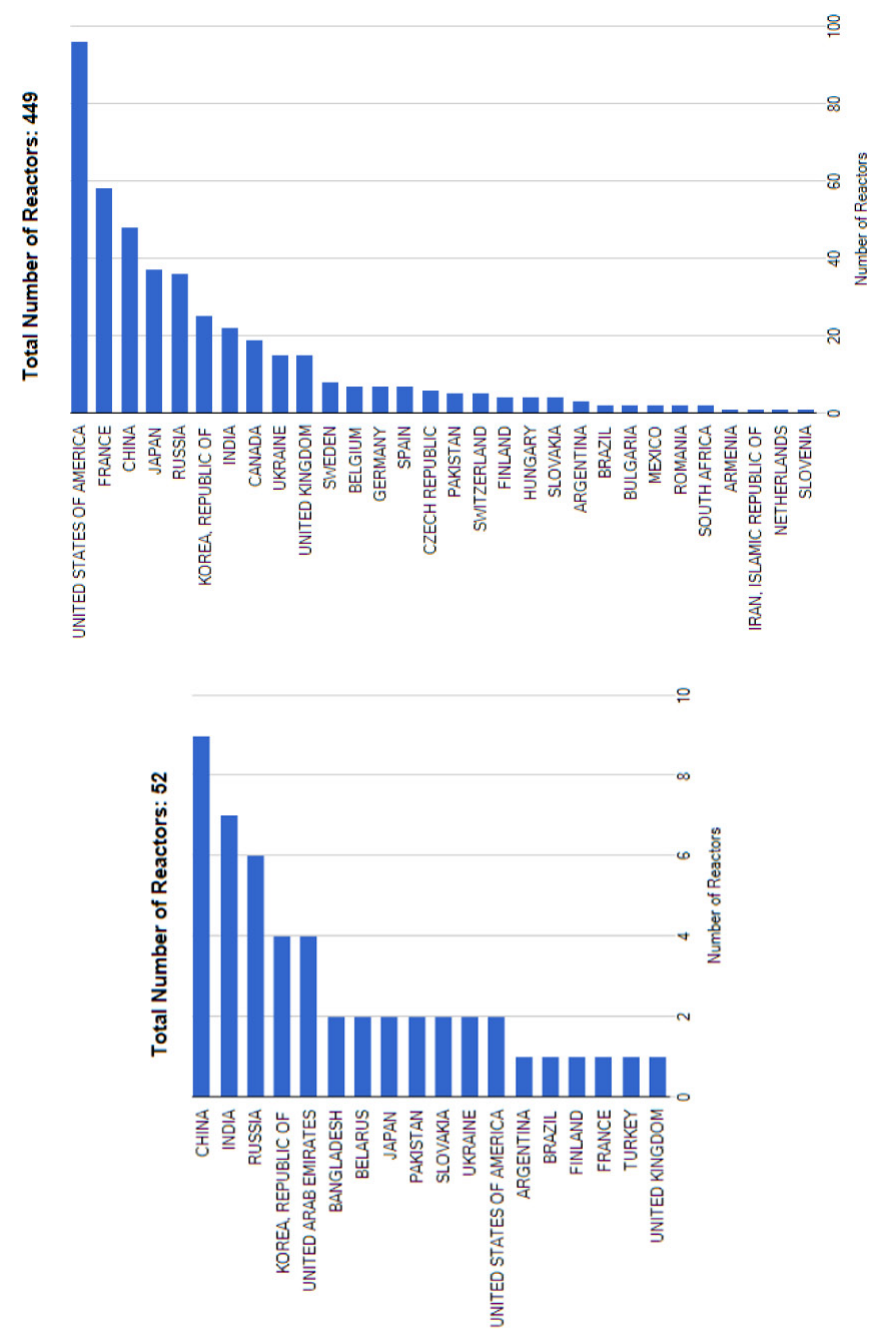

Fig. 7. - Number of operational and long-term shutdown reactors by type (top, as of October 2019). Reactors under construction by type (bottom, as of October 2019) [6] (Copyright (C) 2019 International Atomic Energy Agency (IAEA)).

large part of the cost distribution is below. When comparing the nuclear LCOE median with renewable energies in all discount rate scenarios, it appears cheaper than most, if not all, of them. A similar study was performed by the Intergovernmental Panel on Climate Change (IPCC) [9], that assumed a 10\% discount rate scenario, two scenarios for the number of operational hours of the plants and also two scenarios for the cost of carbon emissions for the $\mathrm{CO}_{2}$ emitting technologies. This study shows that nuclear energy features an interesting LCOE, especially when carbon emission costs are factored in, and is cheaper than many renewable or frontier technologies. 


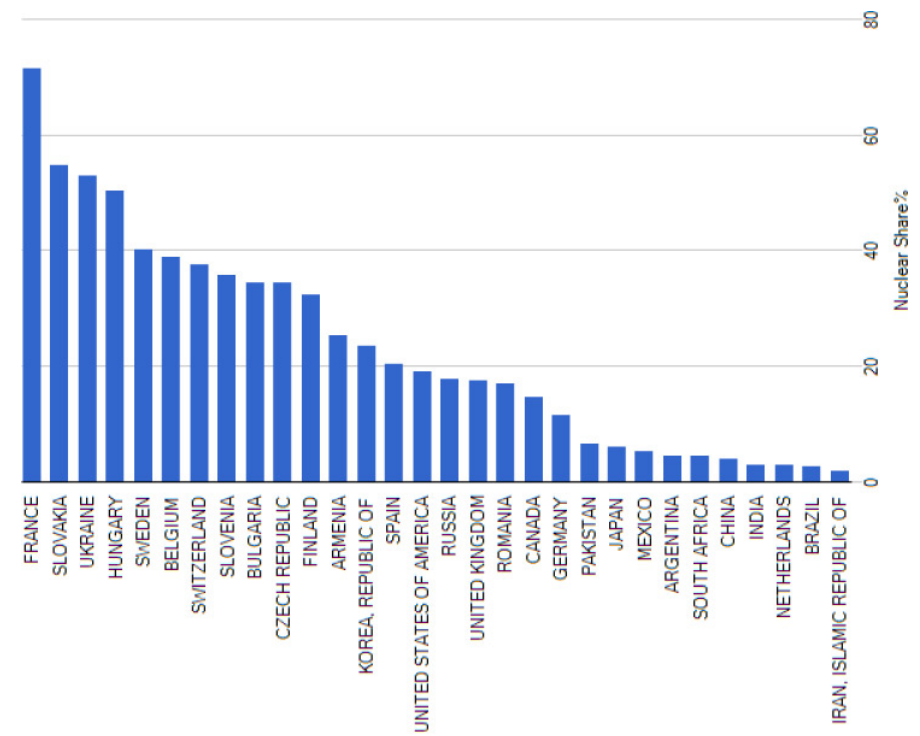

Fig. 8. - Nuclear share of electricity generation in each country (as of October 2019). Note: The share for Taiwan, China was 11.4\% [6]. (Copyright (C) 2019 International Atomic Energy Agency (IAEA)).

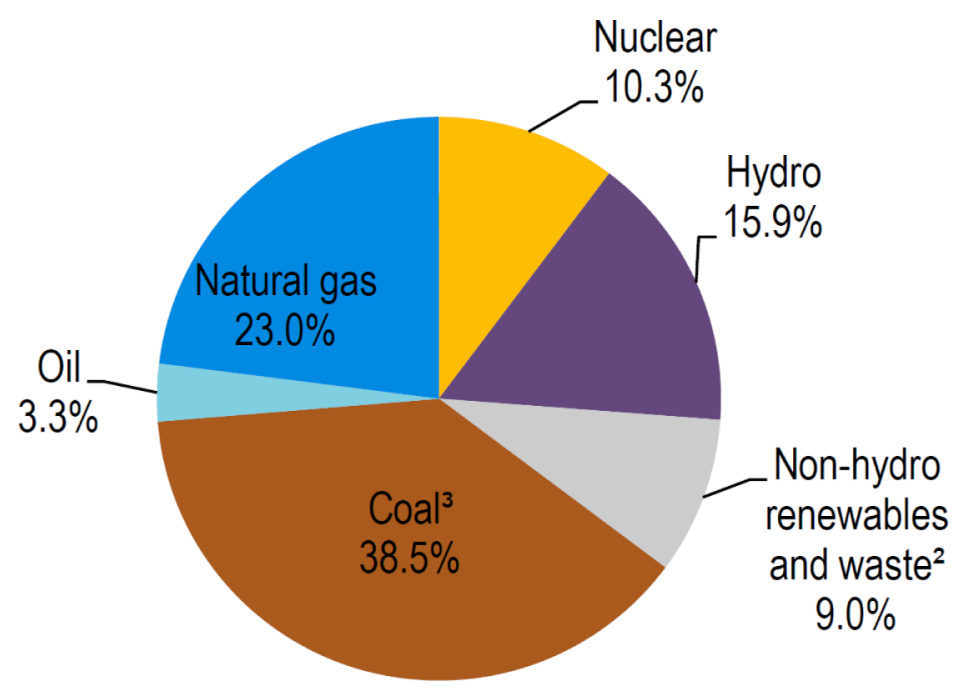

Fig. 9. - World electricity generation (2017) [7] (C) OECD/IEA 2019). 
As far as carbon and other greenhouse gas emissions are concerned, for nuclear energy from fission an accurate estimate of the $\mathrm{CO}_{2}$ equivalent emissions should include all activities in the whole plant lifetime, from fuel mining/extraction to the final decommissioning of the plant. Such a detailed study and comparison with other energy sources in terms of $\mathrm{CO}_{2}$ per KWh produced energy has been performed for instance by the IPCC [9]. Their report clearly shows that a nuclear plant does not produce $\mathrm{CO}_{2}$ or other greenhouse gases during operation, while a small amount is produced as a result of the whole cycle from mining, construction and decommissioning, much less than fossil fuel-fired power plants, actually comparable with many renewables and somewhat less than solar photovoltaic.

\section{5. - Perspectives and issues}

5·1. Nuclear power in future scenarios. - Considering the capability to deploy a large quantity of electric power per plant (typically a power of order 1 GWe with high availability) and the relatively low associate emission level per $\mathrm{kWh}$, it is clear why nuclear energy is still considered one of the players in providing the so-called baseload electricity production, i.e. that minimum quantity of electricity that human activities need independently of the availability of renewable sources, while at the same time meeting greenhouse gas reduction targets. Indeed the IEA projections [7] until 2040, both in the so-called New Policies Scenario and in the Sustainable Development Scenario, nuclear power production is expected to grow.

5.2. Resources. - However, an important point to consider is that of available resources. In other words, we may wonder how much uranium is consumed by the nuclear reactor fleet worldwide and how long will uranium resources last. This is clearly an important question for projecting the use of nuclear power from fission to the future. World annual uranium requirements amounted to 61,600 tU (tons uranium) in 2012 [10]. The so-called identified resources appear to be sufficient for over 120 years, based on the 2012 uranium consumption [10]. Consideration of the entire amount of conventional resources would increase the time scale to well over 300 years, but this would require significant efforts in securing all resources for an effective use. Moving to advanced technology reactors [4] and recycling fuel could increase the long-term availability of nuclear energy from hundreds to thousands of years. In addition, thorium, which is more abundant than uranium in the earth's crust, is another potential source of alternative fuel.

5*3. Obsolescence of the reactor fleet and new construction costs. - One of the issues that nuclear power is facing nowadays is that of the ageing reactor fleet. By looking at the age distribution of reactors in the world, it is evident that most reactors in operation today have been built between the 70 s and the 80 s [11]. Since the lifetime of a plant ranges between 40 and 60 years, depending on each country's specific policy and since on top of that a few countries decided to phase out nuclear power, as a consequence many 
plants are already being decommissioned or will be in the next couple of decades. The question is then whether they will be replaced by new builds or not. Regarding the latter aspect, the nuclear industry is facing a difficult time in terms of the capital investments needed for building new plants, as well as in terms of the actual prices of electricity that utilities need to apply to remain profitable and stay on the market. Indeed, when looking at overnight construction costs of nuclear power capacity (i.e. the cost of a construction project if no interest was incurred during construction, as if the project was completed "overnight"), based on recent US and Western Europe experience, it turns our that such costs have been increasing from about $2000 \mathrm{USD} / \mathrm{KWh}$ in 2005 to between about 7000 and $8000 \mathrm{USD} / \mathrm{KWh}$ in recent years [11]. Moreover, for example in the US nuclear power is experiencing a strong market pressure due to low wholesale electricity prices, driven by low gas prices and rising renewable power capacity (see for instance [12]).

$\mathbf{5}$ 4. Radioactive waste. - We saw in the preceding sections that a nuclear reactor is producing a significant amount of radioactive substances, due to fission itself, as well as due to neutron capture in the fuel and in structural elements. Fission products with a half life until several days are responsible for the large amount of decay heat that is produced within the core immediately after reactor shutdown, i.e. after stopping the chain reaction. However, their radioactivity dies away in a few days or weeks, which means that in case of accidental release of radioactive products they pose a threat only for such a limited amount of time and when the spent fuel is stored in the cooling pools they do not produce anymore heat after a relatively short amount of time. Other ShortLived Fission Products with half lives until a few years can instead be a safety concern also after an accordingly longer period of time, as are Medium-Lived Fission Products with half lives until about 100 years (e.g., ${ }^{137} \mathrm{Cs}$ and ${ }^{90} \mathrm{Sr}$, with half-life of about 30 years). Such isotopes contribute to heat production in the spent fuel and can be a safety concern for decades if accidentally released into the environment. Finally, Long-Lived Fission Products (LLFP) have half-lives over about 200000 years (no fission product has half-life between about a 100 years and 200000 years). Transuranics, i.e. plutonium together with the so-called Minor Actinides, MA, neptunium, americium, curium, etc., decay predominantly by $\alpha$ decay and have half-lives ranging from a few hundreds of years up to above 2 million years $\left({ }^{237} \mathrm{~Np}\right)$. The typical inventory from a 1 GWe PWR fuelled with about 27 tons of $\mathrm{U}\left(3.5 \%{ }^{235} \mathrm{U}\right)$ at discharge (when the spent fuel is removed from the core) is approximately $280 \mathrm{~kg}$ of ${ }^{235} \mathrm{U}, 266 \mathrm{~kg}$ of $\mathrm{Pu}$ of which $56 \mathrm{~kg}$ of fissile ${ }^{239} \mathrm{Pu}, 20 \mathrm{~kg}$ of MA, $13 \mathrm{~kg}$ of ${ }^{90} \mathrm{Sr}$ and $30 \mathrm{~kg}$ of ${ }^{137} \mathrm{Cs}$ (both with 30 years half-life), and $63 \mathrm{~kg}$ of LLFP [13]. The very long decay time of both LLFP and MA is the reason why disposal of spent fuel and similar waste requires very long-term storage, for which one possible solution envisaged is storage deep underground in the so-called geological repositories. Currently, Finland, Sweden and France have advanced projects for building such repositories for final disposal. Some countries apply reprocessing of the spent fuel, where plutonium is extracted from the fuel rods and used to produce new fuel containing both uranium and plutonium, so that the spent fuel is directed to the underground repository only after partial recycling. 
5.5. Waste incineration. - In the effort to improve the safety, security and efficiency of nuclear plants, new concepts of reactors have been developed with goals including the minimization of the production of MAs, a better and more efficient use of the fuel, a better thermodynamic efficiency, the possible production of hydrogen at high temperatures and finally improved safety features to minimize the risk of accidents. All these various concepts are considered within the so-called Generation IV reactors, which are the subject of an international initiative $([4,14])$. As an example, we saw that in a fast reactor, by using e.g. a liquid metal or gas as coolant, the energy spectrum of the neutrons is harder. In such a reactor core, it becomes possible to burn not only fissile elements like ${ }^{235} \mathrm{U}$ and ${ }^{239} \mathrm{Pu}$, but also fissionable elements like ${ }^{238} \mathrm{U}$ for which fission occurs significantly only above a certain energy threshold around $0.5 \mathrm{MeV}$, as can be seen in fig. 3. Although given the much lower fission cross section typically the fuel has to be richer in fissile content, this means that also ${ }^{238} \mathrm{U}$ can to some extent be considered a fuel as well, which has obvious implications in terms of how long uranium resources will last. Another important aspect in fast neutron systems is that, for energies, say, above $0.5 \mathrm{MeV}$, fission becomes dominant over capture, as can again be seen in fig. 3. This means that the MA production, which occurs namely via capture, is suppressed. On the other hand, in a fast reactor the MA produced can be partly destroyed in the reactor itself by fission. An important physics aspect when considering fission of MA is that the amount of delayed neutrons produced upon their fission is significantly less than for ${ }^{235} \mathrm{U}$ and ${ }^{239} \mathrm{Pu}$. This means that for instance fueling a reactor purely with MA is not possible, as one would lose the crucial safety feature of the reactor response times being dominated by the relatively long delayed neutron emission times. However, for Accelerator Driven Systems (ADS) based on a subcritical reactor core that requires an external neutron source, the role of delayed neutrons is not so crucial and therefore there is the possibility to mix a larger quantity of MA into the fuel, thereby offering the possibility to use the apparatus to perform transmutation of the MA into fission products and reduce the radiotoxicity of the final materials in the fuel. This is called PET, partitioning and transmutation approach, meaning that first some nuclides are separated from the spent fuel (partitioning) and then they are irradiated in a special core to be either destroyed or transformed into stable elements (transmutation $[13,15]$ ).

$\mathbf{5}$. Accidents. - A lot of public concern has been raised by nuclear accidents, most notably the ones at Chernobyl in 1986 and Fukushima in 2011. From the previous sections, it is clear that nuclear power must be managed and regulated with maximum care, given the potential impact of accidents entailing release of the radioactive substance inventory into the environment. Even though no industrial activity (and actually no human activity in general) can be made totally risk-free (and indeed several accidents sadly happened for instance in the fossil fuel sector, from oil spills in the sea to fuel truck and train carriage explosions), the goal of nuclear plant designers is to incorporate as many safety measures as possible to prevent any anomaly from escalating to significant nuclear accidents. At the same time, the role of regulators is essential, as independent authorities whose mandate is to check all steps of the nuclear industry from plant design 
through plant operation to interim storage and final disposal of the waste. Last, but not least, research specifically focused on the safety of nuclear plants is conducted worldwide, to pin down and address residual weaknesses, taking advantage of lessons learned from the past plant operational history.

\section{REFERENCES}

[1] Romanelli F., "Fusion energy", these Proceedings.

[2] Java-based Nuclear Data Information System: http://www.oecd-nea.org/janis/.

[3] Classification of Radioactive Waste, No. GSG-1, General Safety Guide (IAEA) 2004, https://www-pub.iaea.org/MTCD/Publications/PDF/Pub1419_web.pdf.

[4] Caciuffo R., "Generation IV nuclear reactor systems", these Proceedings.

[5] Nuclear Energy Today, second edition, NEA No. 6885 (OECD) 2012, p. 9, http://www . oecd-nea.org/pub/nuclearenergytoday/6885-nuclear-energy-today.pdf.

[6] IAEA Power Reactor Information System (PRIS): https://www.iaea.org/PRIS/ home.aspx.

[7] OECD/IEA, 2019 Key World Energy Statistics (IEA Publications) 2019, https:// webstore.iea.org/key-world-energy-statistics-2019, License: http://www.iea.org/ t\&c.

[8] OECD/IEA and OECD/NEA, 2015 Projected Costs of Generating Electricity, 2015 edition (IEA) 2015, pp. 14-15, https://www.oecd-nea.org/ndd/pubs/2015/7057-proj-costselectricity-2015.pdf.

[9] Working Group III Contribution to the Fifth Assessment Report of the Intergovernmental Panel on Climate Change (IPCC) (Cambridge University Press, Intergovernmental Panel on Climate Change) 2014, p. 71.

[10] Uranium 2014: Resources, Production and Demand, NEA No. 7209 (OECD) 2014, http://www . oecd-nea.org/ndd/pubs/2014/7209-uranium-2014.pdf.

[11] OECD/IEA, 2019 Nuclear Power in a Clean Energy System (IEA Publications) 2019, https://webstore.iea.org/nuclear-power-in-a-clean-energy-system, License: http://www.iea.org/t\&c.

[12] "Scientists warn 20\% of US nuclear capacity at risk", in Nuclear Energy Insider, Reuters Events, November 14, 2018 (Nuclear Energy Insider) 2019.

[13] Nifenecker H., Meplan O. and David S., Accelerator Driven Subcritical Reactors (IOP, Bristol and Philadelphia) 2003.

[14] Generation IV International Forum (Physics Publishing, Bristol and Philadelphia) 2003, https://www.gen-4.org/.

[15] Aїт AbDerrahim H., "Realisation of a new large research infrastructure in Belgium, MYRRHA", these Proceedings. 